\title{
Relapse rates in patients with rheumatoid arthritis in stable remission tapering or stopping antirheumatic therapy: interim results from the prospective randomised controlled RETRO study
}

\author{
Judith Haschka, ${ }^{1,2}$ Matthias Englbrecht, ${ }^{1}$ Axel J Hueber, ${ }^{1}$ Bernhard Manger, ${ }^{1}$ \\ Arnd Kleyer, ${ }^{1}$ Michaela Reiser, ${ }^{1}$ Stephanie Finzel, ${ }^{1}$ Hans-Peter Tony, ${ }^{3}$ \\ Stefan Kleinert, ${ }^{3,4}$ Martin Feuchtenberger, ${ }^{3,5}$ Martin Fleck, ${ }^{6}$ Karin Manger, $^{7}$ \\ Wolfgang Ochs, ${ }^{8}$ Matthias Schmitt-Haendle, ${ }^{8}$ Joerg Wendler, ${ }^{4}$ Florian Schuch, ${ }^{4}$ \\ Monika Ronneberger ${ }^{4}$ Hanns-Martin Lorenz, ${ }^{9,10}$ Hubert Nuesslein, ${ }^{11}$ Rieke Alten, ${ }^{12}$ \\ Winfried Demary, ${ }^{13}$ Joerg Henes, ${ }^{14}$ Georg Schett, ${ }^{1}$ Juergen Rech ${ }^{1}$
}

\begin{abstract}
Handling editor Tore K Kvien
- Additional material is published online only. To view please visit the journal online (http://dx.doi.org/10.1136/ annrheumdis-2014-206439).

For numbered affiliations see end of article.

Correspondence to Professor Georg Schett, Department of Internal Medicine 3, Rheumatology and Immunology, University Clinic of Erlangen-Nuremberg, Ulmenweg 18, Erlangen 91054, Germany; georg.schett@uk-erlangen.de
\end{abstract}

Received 8 August 2014 Revised 23 November 2014 Accepted 13 January 2015

Published Online First

6 February 2015

\section{ABSTRACT}

Objective To prospectively analyse the risk for disease relapses in patients with rheumatoid arthritis (RA) in sustained remission, either continuing, tapering or stopping disease-modifying antirheumatic drugs (DMARDs) in a prospective randomised controlled trial.

Methods Reduction of Therapy in patients with Rheumatoid arthritis in Ongoing remission is a multicentre, randomised controlled, parallel-group phase 3 trial evaluating the effects of tapering and stopping all conventional and/or biological DMARDs in patients with RA in stable remission. Patients (disease activity score 28 (DAS28) $<2.6$ for least 6 months) were randomised into three arms, either continuing DMARDs (arm 1), tapering DMARDs by $50 \%$ (arm 2) or stopping DMARDs after 6 months tapering (arm 3). The primary endpoint was sustained remission during 12 months.

Results In this interim analysis, the first 101 patients who completed the study were analysed. At baseline, all patients fulfilled DAS28 remission and 70\% also American College of Rheumatology- European League Against Rheumatism Boolean remission. 82.2\% of the patients received methotrexate, $40.6 \%$ biological DMARDs and $9.9 \%$ other DMARDs. Overall, 67 patients $(66.3 \%)$ remained in remission for 12 months, whereas 34 patients (33.7\%) relapsed. The incidence of relapses was related to study arms ( $p=0.007$; arm 1: $15.8 \%$; arm 2: 38.9\%; arm 3: 51.9\%). Multivariate logistic regression identified anticitrullinated protein antibodies (ACPA) positivity $(p=0.038)$ and treatment reduction (in comparison to continuation) as predictors for relapse (arm 2: $p=0.012 ;$ arm 3: $p=0.003$ ).

Conclusions This randomised controlled study testing three different treatment strategies in patients with RA in sustained remission demonstrated that more than half of the patients maintain in remission after tapering or stopping conventional and biological DMARD treatment. Relapses occurred particularly in the first 6 months after treatment reduction and were associated with the presence of ACPA.

Trial registration number 2009-015740-42.

\section{INTRODUCTION}

Rheumatoid arthritis (RA) is an inflammatory joint disease characterised by synovitis, joint destruction and impaired physical function. ${ }^{1}$ Diagnosis of RA affords the fast initiation and consequent continuation of disease-modifying antirheumatic drugs (DMARDs) treatment. These drugs mitigate or even abrogate the clinical symptoms of RA and prevent damage. Remission of disease is the primary goal in the treatment of RA. ${ }^{2}$ Several factors have substantially improved the outcome of patients with RA during the last years. Hence, (i) $\mathrm{RA}$ is diagnosed earlier, allowing faster treatment initiation; (ii) better monitoring of disease activity has allowed a more flexible treatment adaptation to reach remission; and (iii) biological DMARDs permit the successful treatment of patients with RA failing on conventional drugs.

Based on these developments, an increasing number of patients reach clinical disease remission. A recent survey from the NOR-DMARD study shows doubling of remission rates in the last decade. $^{3}$ Furthermore, many of these patients maintain remission over two subsequent visits. ${ }^{4}$ Due to these changes, new challenges for both physicians and patients arise: patients inquire whether continuation of DMARDs is still necessary or whether treatment can be tapered or even stopped. Paucity of prospective clinical studies, specifically addressing this topic, however, makes it challenging to answer this question. The potential side effects of antirheumatic drugs and their high costs make such considerations even more important. Furthermore, a state of sustained remission implies the dilemma whether disease is actually cured or just effectively suppressed by treatment. Unfortunately, these two states can hardly be distinguished by the currently available clinical tools, which have been successfully developed for monitoring disease activity.

Virtually no prospective randomised controlled trials with the primary aim to taper or stop treatment in patients in sustained disease remission have 
been carried out. The only prospective randomised controlled trials with relapse of disease after treatment tapering as a primary endpoint go back decades. ${ }^{5} 6$ Later trials were either not randomized ${ }^{7-12}$ or were post hoc analyses in randomised clinical trial aiming to achieve remission. ${ }^{13-15}$ Nonetheless, these studies have substantially widened our horizon in the management of RA, suggesting that tapering of antirheumatic treatment may be a feasible strategy in some patients. Also, EULAR guidelines mention the possibility of tapering of biological DMARD therapy once remission is sustained. ${ }^{16}$

To study the potential to taper or stop DMARD therapy, we setup a prospective randomised controlled study, enrolling patients with RA in sustained remission to three different treatment arms, based on continuation, tapering or stopping of DMARD therapy. The primary endpoint of this Reduction of Therapy in patients with Rheumatoid arthritis in Ongoing remission (RETRO) study was the maintenance of remission for 12 months. In addition, we searched for potential predictors for relapses in patients tapering or stopping DMARD treatment.

\section{METHODS}

\section{Study objective and inclusion criteria}

RETRO is an investigator-initiated, phase 3, multicentre, randomised, open, prospective, controlled, parallel-group study (EudraCT number 2009-015740-42). The primary objective was to evaluate the possibility of tapering or stopping antirheumatic therapy in patients fulfilling the American College of Rheumatology (ACR)/European League Against Rheumatism (EULAR) 2010 classification criteria for $\mathrm{RA}^{17}$ and having achieved stable remission. To be enrolled, patients had to have RA for at least 12 months and sustained clinical remission with a disease activity score 28 (DAS28) based on erythrocyte sedimentation rate (ESR) of $<2.6$ for at least 6 months. ${ }^{18}$ Remission had to be documented at least at three sequential visits covering a screening period of 6 months. In addition, patients had to receive stable treatment with antirheumatic drugs, such as conventional DMARDs (methotrexate, leflunomide, hydroxychloroquine and sulfasalazine) and/or biological DMARDs (infliximab, adalimumab, etanercept, golimumab, certolizumab, tocilizumab) without alteration in dose for at least 6 months. Patients receiving rituximab or abatacept or those with steroids of $>5 \mathrm{mg} /$ day within 12 months prior to screening were excluded from the study.

\section{Patients and treatment}

Patients meeting the inclusion criteria and consenting to the study were randomised into three different trial arms: arm 1 (continuation) kept existing conventional and/or biological DMARD regimen at full dose for 12 months. Arm 2 (tapering) reduced the dose of all conventional and/or biological DMARDs by $50 \%$ for the next 12 months. Arm 3 (stop) reduced their dose by $50 \%$ for the first 6 months before entirely stopping all DMARDs (figure 1). Tapering of conventional DMARDs and tocilizumab was done by reducing their respective doses by $50 \%$ without changing application intervals. Tapering of tumour necrosis factor (TNF) inhibitors was accomplished by doubling the time between applications. If stable doses of low-dose glucocorticoids ( $\leq 5 \mathrm{mg}$ /day) were used at baseline, they were also reduced like conventional DMARDs. Non-steroidal anti-inflammatory drugs could be taken on demand before and after inclusion into the study. Relapse of disease was defined as leaving DAS28 remission corresponding to a DAS28-ESR score of $>2.6$. In case of relapse, all patients randomised to the reduction groups were re-exposed to their original full-dose treatment regimen and entered the observational phase of the study. This strategy was predefined in the study protocol.

From May 2010 until May 2013, 101 patients at 11 different centres were randomised and completed the study. Also, 2 of the 101 patients fulfilling the inclusion criteria showed a slightly elevated DAS28 score (2.68 and 2.71) due to upper respiratory tract infection and associated elevation of ESR. Since no increased disease activity of arthritis was found, these two patients were also randomised.

\section{Efficacy assessments}

Primary efficacy parameter was disease activity, which was assessed at baseline and after months 3, 6, 9 and 12 using the DAS28-ESR by designated doctors at each centre. Additionally, fulfilment of the ACR/EULAR Boolean remission criteria was

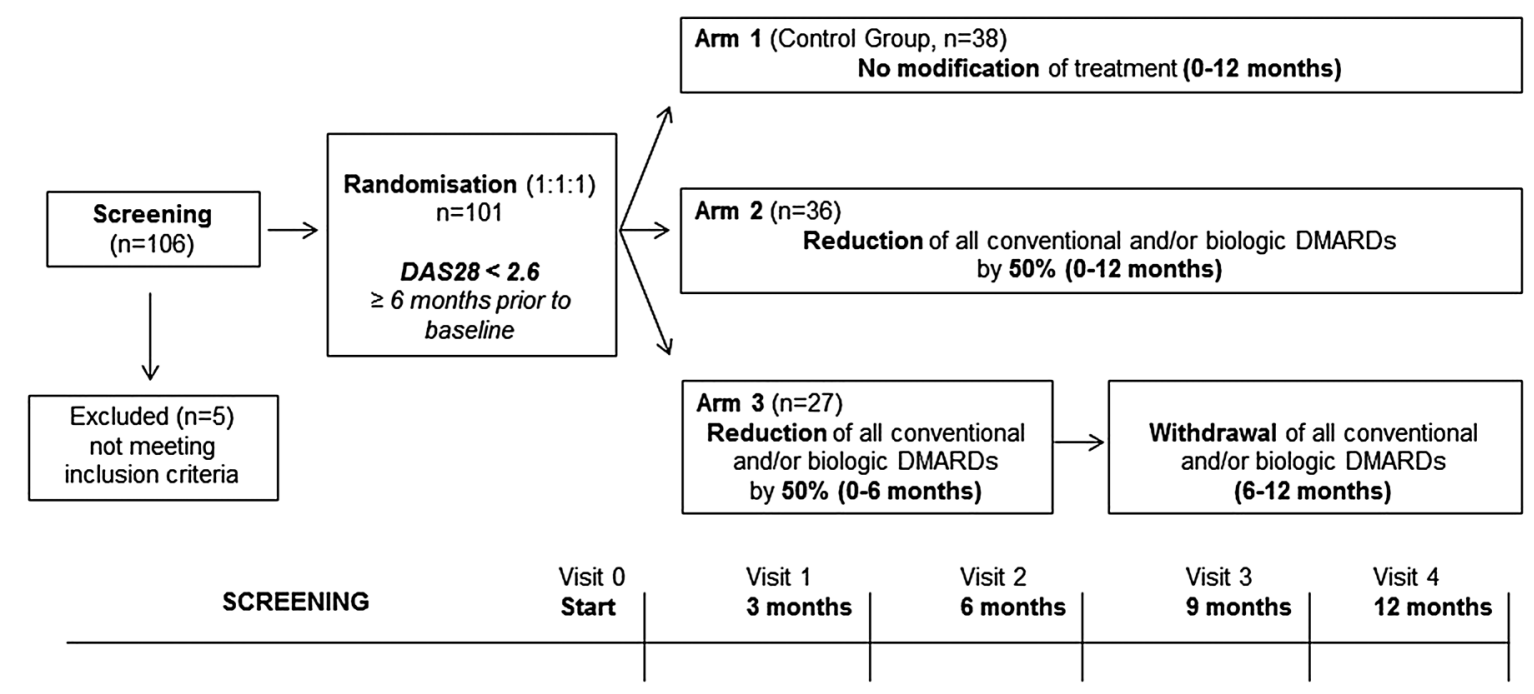

Figure 1 Design of the study. Prospective randomised controlled trial with three treatment arms. In total, 106 patients with rheumatoid arthritis were screened, 101 patients with a disease activity score 28 (DAS28) of $<2.6$, indicating disease remission were randomised into the three study arms (continuation, tapering, stopping) and were followed for 1 year with regular visits every 3 months. DMARDs, disease-modifying antirheumatic drug. 
assessed at each visit. ${ }^{19}$ Assessment of physical function was done using the disability index of the health assessment questionnaire (HAQ-DI). ${ }^{20}$ With respect to demographic and additional disease-specific parameters, age, sex and body mass index, as well as disease duration, duration of remission, rheumatoid factor (RF) and anticitrullinated protein antibodies (ACPA) status, were assessed at baseline. Furthermore, antirheumatic therapy and any other concomitant treatment were recorded at each visit.

\section{Statistical analysis}

We performed an interim analysis of the first 101 patients who completed the 12-month study period. Patients were allocated to treatment arms in a 1:1:1 ratio according to a randomisation plan using randomly permuted blocks (number of blocks: 1) generated by the statistician. According to an a priori sample size calculation, the total study population would be 318 . In descriptive analyses, treatment arms as well as patients in sustained remission versus those relapsing were analysed for demographic and disease-related parameters. Descriptive results are stated in medians and IQRs as well as arithmetic means and SDs. Corresponding inferential comparisons of subgroups were calculated using Kruskal-Wallis or Mann-Whitney U tests for numerical variables and exact $\chi^{2}$ tests for nominal characteristics. A multivariate logistic regression model using 'enter' method was used in order to predict the occurrence of disease relapses from the following set of baseline characteristics including an intercept term: age, gender, duration of disease, duration of remission, RF status, ACPA status, ACR/EULAR remission status, biological DMARD use and allocation to study arms 2 or 3 (in comparison to reference arm 1). Kaplan-Meier plots were used to illustrate relapses over the 12 months of the study with respect to (i) allocation to study arms, (ii) fulfilment of ACR/ EULAR Boolean remission, (iii) ACPA positivity and (iv) treatment with biological DMARDs. SPSS software V.21 was used for calculation of results, whereas $p$ values $\leq 0.05$ were considered statistically significant.

\section{RESULTS}

\section{Baseline characteristics of the patients}

Table 1 summarises the baseline characteristics of the patients. In brief, $61.4 \%$ of the 101 patients were women $(\mathrm{N}=62$ women, $\mathrm{N}=39$ men). Median (IQR) age was 57.0 (19) years while median (IQR) disease duration was 5.0 (7) years. Median duration of remission was 12.0 (12) months before study entry, with no difference between the three trial arms. Swollen and tender joints were very rare in the study population attributing to a median (IQR) swollen joint count of $0.0(0)$ and tender joint count of $0.0(0)$, which was also reflected by more than two-thirds of the patients fulfilling the ACR/EULAR remission criteria $(77.2 \% ; \mathrm{N}=78)$. Importantly, there were no differences in baseline DAS28 scores or the number of patients fulfilling ACR/EULAR remission criteria between the three arms. Also, baseline functional status, measured by HAQ-DI, was generally low and not significantly different among the different treatment arms. The majority of patients were receiving methotrexate (82.2\%), whereas $9.9 \%$ were treated with other conventional DMARDs. Biological DMARDs were used by $40.6 \%$ of the patients with TNF inhibitors as the leading agents (75.6\%).

\section{Prevalence of disease relapses after continuation, tapering or stopping treatment}

In total, 67 of the 101 patients (66.3\%) remained in remission over the entire follow-up period of 12 months, whereas 34 patients $(33.7 \%)$ relapsed (figure 2A). Prevalence of disease relapse was as low as $15.8 \%$ with continuation of antirheumatic therapy ( $\operatorname{arm} 1$ ) but higher when tapering treatment ( $\operatorname{arm~2:}$ $\left.38.9 \% ; \chi^{2}(1)=5.0, p=0.036\right)$ or when stopping it $(\operatorname{arm} 3$ :

Table 1 Baseline characteristics of the patients

\begin{tabular}{|c|c|c|c|c|c|}
\hline Characteristics & $\begin{array}{l}\text { Total population } \\
(n=101)\end{array}$ & $\begin{array}{l}\text { Continuation } \\
\text { Arm } 1 \\
(n=38)\end{array}$ & $\begin{array}{l}\text { Tapering } \\
\text { Arm } 2 \\
(n=36)\end{array}$ & $\begin{array}{l}\text { Stopping } \\
\text { Arm } 3 \\
(n=27)\end{array}$ & p Values \\
\hline Age, years & $57.0(19)$ & $59.0(20)$ & 56.5 (18) & $56.0(21)$ & 0.977 \\
\hline Female gender, $\%(\mathrm{~N})$ & $61.4(62)$ & $52.6(20)$ & $61.1(22)$ & $74.1(20)$ & 0.219 \\
\hline Disease duration, years & $5.0(7)$ & $5.5(7)$ & $6.0(6)$ & $3.0(5)$ & 0.048 \\
\hline Remission duration, months & $12.0(12)$ & $12.0(27)$ & $9.0(12)$ & $12.0(10)$ & 0.126 \\
\hline DAS28, Units & $1.91(0.85)$ & $1.8(1.0)$ & $1.9(0.8)$ & $2.0(0.8)$ & 0.464 \\
\hline ACR/EULAR remission, \% (N) & $77.2(78)$ & $78.9(30)$ & $75.0(27)$ & $77.8(21)$ & 0.790 \\
\hline Tender joint count $28, \mathrm{~N}$ & $0.0(0)$ & $0.0(0)$ & $0.0(0)$ & $0.0(0)$ & 0.351 \\
\hline Swollen joint count $28, \mathrm{~N}$ & $0.0(0)$ & $0.0(0)$ & $0.0(0)$ & $0.0(0)$ & 0.740 \\
\hline VAS patient global, mm & $0.0(6)$ & $0.3(8)$ & $0.0(6)$ & $0.0(5)$ & 0.525 \\
\hline $\mathrm{ESR}, \mathrm{mm}$ & $13.0(14)$ & $9.0(16)$ & $13.0(13)$ & $14.0(9)$ & 0.693 \\
\hline CRP, mg/dL & $0.28(0.24)$ & $0.24(0.23)$ & $0.29(0.35)$ & $0.29(0.22)$ & 0.403 \\
\hline HAQ, Units & $0.0(0.25)$ & $0.0(0.13)$ & $0.0(0.25)$ & $0.0(0.19)$ & 0.730 \\
\hline Positive RF, \% (N) & $63.4(64)$ & $55.3(21)$ & $69.4(25)$ & $66.7(18)$ & 0.446 \\
\hline Positive ACPA, \% (N) & $59.4(60)$ & $60.5(23)$ & $55.6(20)$ & $63.0(17)$ & 0.966 \\
\hline Methotrexate Use, \% (N) & $82.2(83)$ & $86.8(33)$ & $69.4(25)$ & $92.6(25)$ & 0.048 \\
\hline Other DMARD* Use, \% (N) & $9.9(10)$ & $7.9(3)$ & $13.9(5)$ & $7.4(2)$ & 0.699 \\
\hline Biological DMARD† Use, \% (N) & $40.6(41)$ & $42.1(16)$ & $50.0(18)$ & $25.9(7)$ & 0.152 \\
\hline \multicolumn{6}{|c|}{$\begin{array}{l}\text { Significant values are shown in bold typeface. } \\
\text { *Leflunomide, sulfasalazine, hydroxychloroquine. } \\
\text { †Tumour necrosis factor inhibitors and tocilizumab; Data show medians and IQRs. } \\
\text { ACPA, anticitrullinated protein antibodies; ACR, American College of Rheumatology; CRP, C-reactive protein; DAS28, disease activity score 28 (based on ESR); DMARDs, } \\
\text { disease-modifying antirheumatic drugs; ESR, erythrocyte sedimentation rate; EULAR, European League Against Rheumatism; HAQ, health assessment questionnaire; RF, rheumatoid } \\
\text { factor; VAS, visual analoque scale. }\end{array}$} \\
\hline
\end{tabular}




\section{Clinical and epidemiological research}
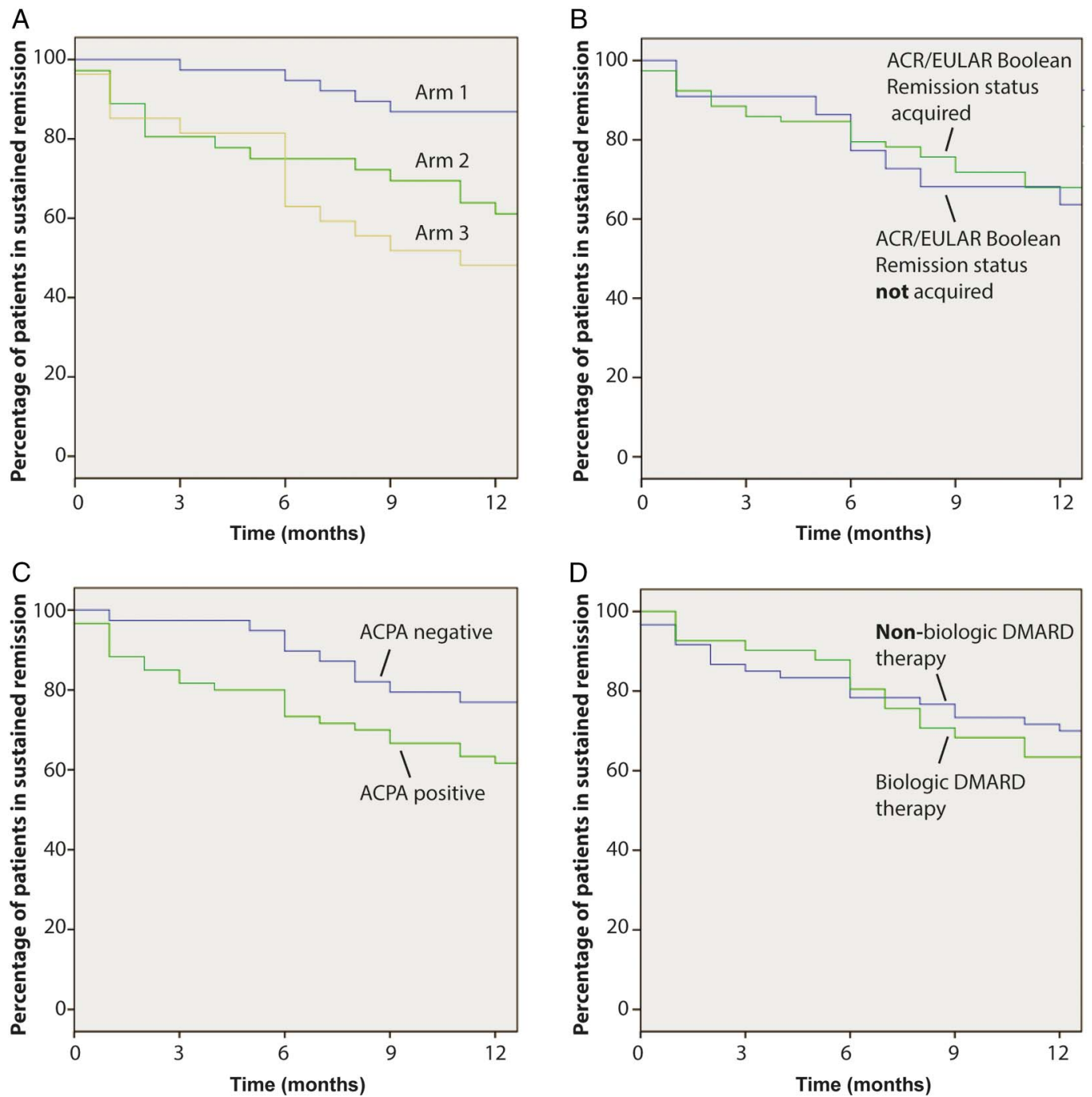

Figure 2 Kaplan-Meier curves for maintenance of remission. Curves indicate loss of remission over 12 months in patients with rheumatoid arthritis in relation to different parameters. (A) Treatment arms (blue: continuing; green: tapering; yellow: stopping). (B) American College of Rheumatology (ACR)/European League Against Rheumatism (EULAR) remission fulfilled (green) or not fulfilled (blue) at baseline. (C) Anticitrullinated protein antibody (ACPA) status (green: positive; blue: negative). (D) Use of biological disease-modifying antirheumatic drugs (DMARDs) therapy (green: use of biological DMARDs; blue: no biological DMARDs). The y-axis indicates the percentage of patients with rheumatoid arthritis in sustained remission (100\% at baseline), and the $x$-axis indicates time.

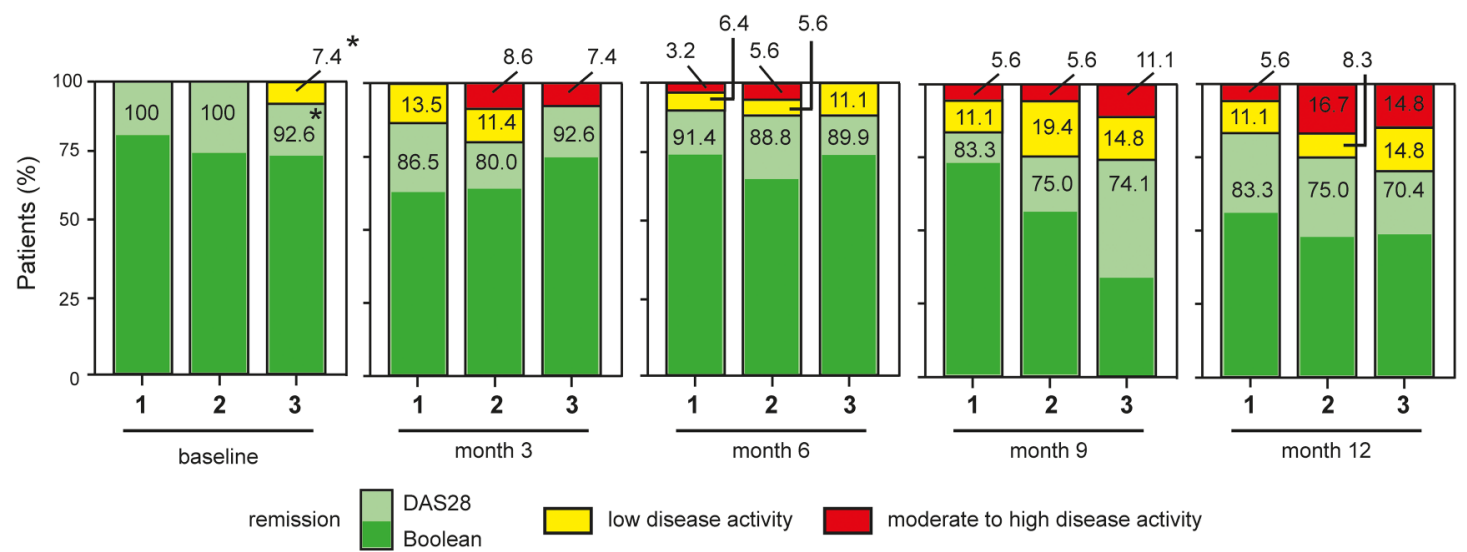

Figure 3 Development of disease activity score 28 (DAS28)-erythrocyte sedimentation rate. Bars indicate percentage of patients in remission (green), low-disease activity (yellow) and moderate-to-high-disease activity (red) in the three study arms (arm 1: continuing; arm 2: tapering; arm 3: stopping) at baseline and months 3, 6, 9 and 12. Remission is defined by DAS28 of <2.6, low-disease activity between 2.61 and 3.2 and moderate-to-high-disease activity represents a DAS28 score of $>3.21$. *Two patients had a DAS28 slightly above 2.6 due to respiratory infection without any clinical signs of activity of arthritis. 
Table 2 Baseline characteristics of patients sustaining remission or relapsing

\begin{tabular}{|c|c|c|c|c|c|c|}
\hline \multirow[b]{2}{*}{ Characteristics } & \multicolumn{3}{|l|}{ Total population } & \multicolumn{3}{|l|}{ ACPA-positive patients } \\
\hline & $\begin{array}{l}\text { Sustained remission } \\
(\mathrm{N}=67)\end{array}$ & $\begin{array}{l}\text { Relapse } \\
(\mathrm{N}=34)\end{array}$ & p Values & $\begin{array}{l}\text { Sustained remission } \\
(\mathrm{N}=36)\end{array}$ & $\begin{array}{l}\text { Relapse } \\
(\mathrm{N}=24)\end{array}$ & $p$ Values \\
\hline Age, years & $59.0(17)$ & $55.0(21)$ & 0.849 & $50.0(16)$ & $52.0(17)$ & 0.608 \\
\hline Female gender, \% (N) & $55.2(37)$ & $73.5(25)$ & 0.087 & $50.0(18)$ & $83.3(20)$ & 0.013 \\
\hline Disease duration, years & $5.0(6)$ & $6.0(7)$ & 0.278 & $5.0(6)$ & $6.0(7)$ & 0.868 \\
\hline Remission duration, months & $11.5(12)$ & $12.0(12)$ & 0.884 & $11.0(20)$ & $13.0(18)$ & 0.925 \\
\hline DAS28, Units & $1.82(1.1)$ & $2.08(0.6)$ & 0.071 & $1.68(1.1)$ & $2.06(0.7)$ & 0.131 \\
\hline ACR/EULAR remission, \% (N) & $77.6(52)$ & $76.5(26)$ & 0.803 & $69.4(25)$ & $83.3(20)$ & 0.361 \\
\hline Tender joint count $28, \mathrm{~N}$ & $0.0(0)$ & $0.0(0)$ & 0.586 & $0.0(0)$ & $0.0(0)$ & 0.496 \\
\hline Swollen joint count $28, \mathrm{~N}$ & $0.0(0)$ & $0.0(0)$ & 0.362 & $0.0(0)$ & $0.0(0)$ & 0.057 \\
\hline VAS patient global, mm & $0.0(6)$ & $0.0(6)$ & 0.583 & $0.5(9)$ & $0.0(5)$ & 0.436 \\
\hline $\mathrm{ESR}, \mathrm{mm}$ & $11.0(13)$ & $15.0(13)$ & 0.080 & $9.0(11)$ & $14.0(14)$ & 0.157 \\
\hline $\mathrm{CRP}, \mathrm{mg} / \mathrm{dL}$ & $0.23(0.3)$ & $0.30(0.4)$ & 0.170 & $0.21(0.3)$ & $0.30(0.3)$ & 0.386 \\
\hline HAQ, Units & $0.0(0.3)$ & $0.0(0.2)$ & 0.847 & $0.0(0.1)$ & $0.0(0.1)$ & 0.714 \\
\hline Positive RF, \% (N) & $59.7(40)$ & $70.6(24)$ & 0.383 & $91.7(33)$ & $83.3(20)$ & 0.422 \\
\hline Positive ACPA, \% (N) & $53.7(36)$ & $70.6(24)$ & 0.126 & - & - & - \\
\hline Methotrexate use, \% (N) & $85.1(57)$ & $76.5(26)$ & 0.409 & $88.9(32)$ & $75.0(18)$ & 0.178 \\
\hline Other DMARD* use, \% (N) & $10.4(7)$ & $8.8(3)$ & 1.000 & $8.3(3)$ & $8.3(2)$ & 1.000 \\
\hline Biological DMARD† use, \% (N) & $37.3(25)$ & $47.1(16)$ & 0.395 & $47.2(17)$ & $45.8(11)$ & 1.000 \\
\hline \multicolumn{7}{|c|}{$\begin{array}{l}\text { Significant values are shown in bold typeface. } \\
\text { *Leflunomide, sulfasalazine, hydroxychloroquine. } \\
\text { †Tumour necrosis factor inhibitors and tocilizumab; data show medians and IQRs. } \\
\text { ACPA, anti-citrullinated protein antibodies; ACR, American College of Rheumatology; CRP, C-reactive protein; DAS28, disease activity score } 28 \text { (based on ESR); DMARDs, } \\
\text { disease-modifying antirheumatic drugs; ESR, erythrocyte sedimentation rate; EULAR, European League Against Rheumatism; HAQ, health assessment questionnaire; RF, rheumatoid } \\
\text { factor; VAS, visual analogue scale. }\end{array}$} \\
\hline
\end{tabular}

$\left.51.9 \% ; \chi^{2}(1)=9.6, \quad \mathrm{p}=0.003\right)$. Overall, 44.4\% $(\mathrm{N}=28)$ of patients in the two reduction arms relapsed. No significant differences regarding the incidence of relapses between the tapering and stopping regimen arms were found $\left(\chi^{2}=1.050\right.$, $\mathrm{p}=0.443)$. The majority of relapses $(\mathrm{N}=19)$ occurred within the first 6 months during the tapering phase ( $\operatorname{arm} 2: \mathrm{N}=9$ out of 14; $\operatorname{arm} 3: \mathrm{N}=10$ out of 14 ) of the study. Figure 3 shows the development of DAS28 values over 12 months of study period.

\section{Baseline characteristics of patients remaining in remission or relapsing}

Descriptive baseline characteristics of total population and ACPA-positive patients who remained in remission or experienced a relapse of disease are depicted in table 2. Age, disease duration and duration of remission at baseline were very similar between the remission and the relapse group. Although ESR levels and DAS28 scores showed a tendency for higher baseline values in patients who relapsed, other single and composite clinical activity measures, such as tender and swollen joint counts, C-reactive protein level and ACR/EULAR remission status, were virtually identical in the two groups, suggesting no major impact of residual disease activity on subsequent relapses. With respect to ACPA status, $>70 \%$ of the patients with relapsing disease showed ACPA positivity, whereas this number was lower (53\%) in patients remaining in continuous remission. Furthermore, relapsing patients were preferentially women (73.5\%), whereas sex distribution in the remission group was similar (55.2\% women). Interestingly, significantly more ACPA-positive patients who flared were women $(50.0 \%$ women vs $83.3 \%, \mathrm{p}=0.013)$.

\section{Predictors for disease relapse}

We next performed a multivariate logistic regression model to define predictors for disease relapse in patients with RA in remission. In a model containing demographic variables (age, sex), autoantibody status (RF, ACPA), remission status (ACR/ EULAR Boolean remission) and other disease-specific variables (disease duration, remission duration, biological DMARD exposure), only ACPA positivity (Wald $\chi^{2}=4.3, p=0.038$, $\mathrm{OR}=5.23)$ and treatment reduction in comparison to continuation as reference $\left(\operatorname{arm} 2\right.$ : Wald $\chi^{2}=6.3, p=0.012, \mathrm{OR}=5.74$, arm 3: Wald $\chi^{2}=8.8, \mathrm{p}=0.003$, $\mathrm{OR}=8.78$ ) were identified as predictors for subsequent relapse of disease (table 3). Female sex showed a tendency for prediction of relapses though not being statistically significant (Wald $\chi^{2}=2.9, p=0.089$ ). The remaining characteristics including fulfilment of Boolean remission criteria did not significantly contribute to prediction quality of the model. Overall correct case classification rate of the model was $71.6 \%$. A post hoc power analysis of the current findings for the weakest significant predictor in multivariate logistic regression (ie, ACPA) suggests a power of $80.3 \%$ upon end of the trial. Similar results were generated with an alternative multivariate logistic regression model where Boolean remission criteria were replaced by baseline ESR since there was no additive value for this parameter in the first model (see online supplementary table S3).

The role of ACPA in disease relapse is also shown in online supplementary figure $S 1$, indicating that remission patients with negative ACPA showed rather low prevalence for relapse independent whether treatment was continued, tapered or stopped. In contrast, ACPA-positive patients in remission showed a gradual increase of relapse rates with respect to study arm allocation, suggesting significance for $\operatorname{arm} 3\left(\chi^{2}(1)=6.45, \mathrm{p}=0.018\right)$ in a bivariate setting comparing loss of remission to ACPA status.

\section{DISCUSSION}

RETRO addresses a novel and growing challenge in RA: due to better treatment, more patients experience disease remission, raising the question whether medication has to be continued 
Table 3 Results of multivariate logistic regression for prediction of relapse

\begin{tabular}{|c|c|c|c|c|c|}
\hline & B & Wald & $\mathrm{p}$ Value & OR & OR $(95 \% \mathrm{Cl})$ \\
\hline Age & 0.028 & 1.638 & 0.201 & 1.029 & 0.985 to 1.074 \\
\hline Sex & 0.951 & 2.885 & 0.089 & 2.588 & 0.864 to 7.756 \\
\hline ACR/EULAR remission & -0.396 & 0.448 & 0.503 & 0.673 & 0.211 to 2.144 \\
\hline Disease duration & 0.007 & 0.029 & 0.864 & 1.007 & 0.926 to 1.096 \\
\hline Remission duration & 0.019 & 0.985 & 0.321 & 1.019 & 0.982 to 1.058 \\
\hline Positive RF & -0.897 & 1.505 & 0.220 & 0.408 & 0.097 to 1.709 \\
\hline Positive ACPA & 1.655 & 4.327 & 0.038 & 5.231 & 1.100 to 24.867 \\
\hline Biological DMARD & 0.502 & 0.775 & 0.379 & 1.652 & 0.540 to 5.049 \\
\hline Continuation versus tapering (arm 2) & 1.747 & 6.297 & 0.012 & 5.737 & 1.466 to 22.454 \\
\hline Continuation versus stopping (arm 3) & 2.172 & 8.751 & 0.003 & 8.777 & 2.081 to 37.013 \\
\hline Intercept & -5.998 & 8.872 & 0.003 & 0.002 & \\
\hline
\end{tabular}

Significant values are shown in bold typeface.

ACPA, anticitrullinated protein antibodies; ACR, American College of Rheumatology; DMARDs, disease-modifying antirheumatic drugs; EULAR, European League Against Rheumatism; $\mathrm{RF}$, rheumatoid factor.

lifelong or can be tapered or stopped. Although remission describes a state with no or very minor disease, it cannot be seen as homogeneous state. Most importantly, remission covers two fundamentally different situations, effective suppression of inflammation and cure, which are hardly distinguishable but differ in their likelihood for disease relapse when treatment is stopped. To answer this important question, strategy studies on tapering and stopping antirheumatic therapies in remission patients are of seminal importance. ${ }^{21-24}$

Tapering and stopping DMARDs becomes an increasing issue for patients and physicians the longer the remission lasts. In the RETRO cohort, mean duration of remission was clearly $>1$ year, suggesting highly stable remission. Nonetheless, 15\% of the patients continuing full-dose DMARDs lost their remission state, indicating that even 'stable' remission is not an entirely fixed condition. The fraction of patients with a DAS28 of $>3.2$, however, was only $5.6 \%$. This situation has to be seen in light of the strict inclusion of the patients requiring stable DAS28 remission of at least 6 months. The overall relapse rate in patients with RA in remission after tapering or stopping antirheumatic treatment was significantly higher (44\%) than in patients continuing therapy. Although higher relapse rates were expected, data also showed that more than half of the patients with RA could successfully taper or stop their treatment. Two-thirds of relapses occurred within the first 6 months, suggesting that appropriate dosing of the DMARD mono therapy or combination therapy was seminal for maintaining remission.

No randomised controlled trials with the primary aim to taper and stop antirheumatic treatment in patients with RA in remission have been carried out to date. RETRO was designed to improve decision making in sustained remission of RA by defining relapse rates and finding predictors for relapse. Thereby, RETRO was applied in all patients with RA irrespective of their previous treatment and tapering was done with all DMARDs used by the individual patient. The later, in the year 2013, published EULAR recommendations for the treatment of RA proposed that tapering of DMARDs should follow a sequential order with glucocorticoids first, followed by biological and then conventional DMARDs. ${ }^{16}$ However, this part of the recommendations is not based on randomised controlled studies comparing different strategies of tapering and stopping these drugs. In fact, such studies are essentially needed to improve these recommendations. Our approach tapering all drugs by $50 \%$ was chosen because it is pragmatic, reflects patients' demands and attributes to the principal question of patients and physicians whether RA is only suppressed by drug therapy or whether it is actually cured.

Previous observational studies addressing tapering and stopping DMARDs have focused on withdrawal of TNF inhibitors $^{7-11} 13-15$ or have studied the subpopulation of patients with early arthritis. ${ }^{13-15} 2425$ Tanaka and colleagues withdrew the TNF inhibitor infliximab in patients with established RA in lowdisease activity. ${ }^{9}$ Of the $81 \%$ of patients in clinical remission at baseline, $47 \%$ remained in remission after 1 year. Despite this very different approach (i) focusing on a selected therapy, (ii) leaving the patients on methotrexate treatment and (iii) using low-disease activity rather than remission as the key outcome parameter, relapse rates are stunningly similar. Furthermore, data from the BeSt and Leiden Early Arthritis Clinic cohorts suggested that drug-free remission is preferentially seen in the absence of ACPA. ${ }^{25-27}$ Indeed, ACPA were the main factor determining the risk for relapse in our study. ACPA precede the onset of RA, ${ }^{28}$ are associated with more severe disease ${ }^{29}$ and trigger pathological changes even in the absence of RA. ${ }^{30-32}$ Hence, the presence of ACPA in patients with RA in remission may indicate an underlying autoimmune process hampering successful treatment withdrawal.

Several other factors can be considered as predictors for disease relapse. One of the factors is remission quality: in this cohort, $77.2 \%$ of patients fulfilled ACR/EULAR Boolean remission criteria, indicating deep remission in the majority of patients. 'Deep' remission, however, was not predictive for remaining in remission after treatment tapering in our study. In the specific case of TNF inhibitor withdrawal, however, remission depth may affect disease relapses, as Tanaka and colleagues have suggested. ${ }^{8}$ Hence, data from the HONOR study investigating withdrawal of adalimumab indicated that patients with a lower DAS28 $(\leq 1.98)$ remained in low-disease activity more frequently than patients with a higher baseline DAS28 (1.98-2.6). ${ }^{8}$ Interestingly, also longer disease duration and the use of biological DMARDs, suggestive of a more resistant phenotype of RA, did not predict a higher relapse rate in our study.

Strengths of the study are its real-life setting with applicability to the vast majority of patients with RA, its design as a prospective randomised controlled trial and its rigorous focus on patients in stable remission. One limitation is the lack of radiographic data, which leaves the possibility that residual disease activity could induce structural damage, although we think that such effects, if 
existent, are at best small. Furthermore, the lack of blinding to the study arms could have influenced outcomes, but has not been feasible in such context. Also, despite baseline characteristics being very evenly distributed, small differences in disease duration and methotrexate use were found between the study arms, but none of them was consistently present between the continuation and the two tapering arms. Finally, we cannot exclude that additional patients experience disease relapses after 12 months.

In summary, the RETRO study gives a new perspective in managing patients with RA having reached stable disease remission. It suggests that tapering and even stopping antirheumatic treatment is feasible and permits more than half of the patients to maintain remission over 1 year. Nonetheless, caution should be undertaken in patients with ACPA showing a higher risk for relapse.

\author{
Author affiliations \\ ${ }^{1}$ Department of Internal Medicine 3, University of Erlangen-Nuremberg, Erlangen, \\ Germany \\ ${ }^{2}$ Department of Internal Medicine 2, The Vinforce Study Group, Saint Vincent \\ Hospital, Vienna, Austria \\ ${ }^{3}$ Department of Internal Medicine 2, University of Wurzburg, Wurzburg, Germany \\ ${ }^{4}$ Rheumatology Practice, Erlangen, Germany \\ ${ }^{5}$ Rheumatology Practice and Department of Internal Medicine 2, Clinic Burghausen, \\ Burghausen, Germany \\ ${ }^{6}$ Department of Rheumatology and Clinical Immunology, Asklepios Medical Center, \\ Bad Abbach, Germany \\ ${ }^{7}$ Rheumatology Practice, Bamberg, Germany \\ ${ }^{8}$ Rheumatology Practice, Bayreuth, Germany \\ ${ }^{9}$ Department of Internal Medicine V, Division of Rheumatology, University of \\ Heidelberg, Heidelberg, Germany \\ ${ }^{10}$ ACURA Center for Rheumatic Diseases, Baden-Baden, Germany \\ ${ }^{11}$ Rheumatology Practice, Nuremberg, Germany \\ ${ }^{12}$ Schlosspark Clinic, Berlin, Germany \\ ${ }^{13}$ Rheumatology Practice, Hildesheim, Germany \\ ${ }^{14}$ Department of Internal Medicine 2, University of Tubingen, Tubingen, Germany
}

Contributors JH, MR, AK, BM, H-PT, SK, MF, MF, KM, WO, MS-H, JW, FS, MR, $\mathrm{H}-\mathrm{ML}, \mathrm{HN}, \mathrm{RA}, \mathrm{WD}$ and JH collected and analysed the data. SF, AJH, JR and GS designed the study. ME performed statistical analyses. JH, JR and GS wrote the manuscript.

Funding This study was supported by the Deutsche Forschungsgemeinschaft (SPP1468-IMMUNOBONE), the Bundesministerium für Bildung und Forschung (BMBF; project METARTHROS), the Marie Curie project OSTEOIMMUNE, the TEAM and MASTERSWITCH projects of the European Union and the IMI funded project BTCure.

Competing interests None.

Patient consent Obtained.

Ethics approval Ethic committee of the Friedrich-Alexander-University of Erlangen-Nuremberg and local ethic committees of the external centres as well as the Paul Ehrlich Institute (PEI) and was conducted according to the ethical principles of the Declaration of Helsinki.

Provenance and peer review Not commissioned; externally peer reviewed.

\section{REFERENCES}

1 Mclnnes IB, Schett $\mathrm{G}$. The pathogenesis of rheumatoid arthritis. N Engl J Med 2011;365:2205-19.

2 Smolen JS, Aletaha D, Bijlsma JW, et al. Treating rheumatoid arthritis to target: recommendations of an international task force. Ann Rheum Dis 2010;69:631-7.

3 Aga $A B$, Lie E, Uhlig $T$, et al. Time trends in disease activity, response and remission rates in rheumatoid arthritis during the past decade: results from the NOR-DMARD study 2000-2010. Ann Rheum Dis 2015;74:381-8.

4 Prince FH, Bykerk VP, Shadick NA, et al. Sustained rheumatoid arthritis remission is uncommon in clinical practice. Arthritis Res Ther 2012;14:R68.

5 ten Wolde S, Breedveld FC, Hermans J, et al. Randomised placebo-controlled study of stopping second-line drugs in rheumatoid arthritis. Lancet 1996;347:347-52.

6 Ahern MJ, Hall ND, Case K, et al. D-penicillamine withdrawal in rheumatoid arthritis. Ann Rheum Dis 1984;43:213-17.

7 Tanaka Y, Hirata S, Kubo S, et al. Discontinuation of adalimumab after achieving remission in patients with established rheumatoid arthritis: 1-year outcome of the HONOR study. Ann Rheum Dis 2015;74:389-95.

8 Hirata S, Saito K, Kubo S, et al. Discontinuation of adalimumab after attaining disease activity score 28-erythrocyte sedimentation rate remission in patients with rheumatoid arthritis (HONOR study): an observational study. Arthritis Res Ther 2013;15:R135.

9 Tanaka Y, Takeuchi T, Mimori T, et al. Discontinuation of infliximab after attaining low disease activity in patients with rheumatoid arthritis: RRR (remission induction by Remicade in RA) study. Ann Rheum Dis 2010;69:1286-91.

10 Saleem B, Keen $H$, Goeb V, et al. Patients with RA in remission on TNF blockers: when and in whom can TNF blocker therapy be stopped? Ann Rheum Dis 2010;69:1636-42.

11 Brocq O, Millasseau E, Albert C, et al. Effect of discontinuing TNFalpha antagonist therapy in patients with remission of rheumatoid arthritis. Joint Bone Spine 2009;76:350-5.

12 Aguilar-Lozano L, Castillo-Ortiz JD, Vargas-Serafin C, et al. Sustained clinical remission and rate of relapse after tocilizumab withdrawal in patients with rheumatoid arthritis. J Rheumatol 2013;40:1069-73.

13 Quinn MA, Conaghan PG, O'Connor PJ, et al. Very early treatment with infliximab in addition to methotrexate in early, poor-prognosis rheumatoid arthritis reduces magnetic resonance imaging evidence of synovitis and damage, with sustained benefit after infliximab withdrawal: results from a twelve-month randomized, double-blind, placebo-controlled trial. Arthritis Rheum 2005;52:27-35.

14 van den Broek M, Klarenbeek NB, Dirven L, et al. Discontinuation of infliximab and potential predictors of persistent low disease activity in patients with early rheumatoid arthritis and disease activity score-steered therapy: subanalysis of the BeSt study. Ann Rheum Dis 2011;70:1389-94.

15 Smolen JS, Emery P, Fleischmann R, et al. Adjustment of therapy in rheumatoid arthritis on the basis of achievement of stable low disease activity with adalimumab plus methotrexate or methotrexate alone: the randomised controlled OPTIMA trial. Lancet 2014;383:321-32.

16 Smolen JS, Landewe R, Breedveld FC, et al. EULAR recommendations for the management of rheumatoid arthritis with synthetic and biological disease-modifying antirheumatic drugs: 2013 update. Ann Rheum Dis 2014;73:492-509.

17 Aletaha D, Neogi T, Silman AJ, et al. 2010 rheumatoid arthritis classification criteria: an American College of Rheumatology/European League Against Rheumatism collaborative initiative. Ann Rheum Dis 2010;69:1580-8.

18 Prevoo ML, van 't Hof MA, Kuper HH, et al. Modified disease activity scores that include twenty-eight-joint counts. Development and validation in a prospective longitudinal study of patients with rheumatoid arthritis. Arthritis Rheum 1995:38:44-8.

19 Felson DT, Smolen JS, Wells G, et al. American College of Rheumatology/European League against Rheumatism provisional definition of remission in rheumatoid arthritis for clinical trials. Ann Rheum Dis 2011;70:404-13.

20 Fries JF, Spitz P, Kraines RG, et al. Measurement of patient outcome in arthritis. Arthritis Rheum 1980;23:137-45.

21 Chatzidionysiou K, van Vollenhoven RF. When to initiate and discontinue biologic treatments for rheumatoid arthritis? J Intern Med 2011;269:614-25.

22 O'Mahony R, Richards A, Deighton C, et al. Withdrawal of disease-modifying antirheumatic drugs in patients with rheumatoid arthritis: a systematic review and meta-analysis. Ann Rheum Dis 2010;69:1823-6.

23 van den Broek M, Huizinga TW, Dijkmans BA, et al. Drug-free remission: is it already possible? Curr Opin Rheumatol 2011;23:266-72.

24 Allaart CF, Lems WF, Huizinga TW. The BeSt way of withdrawing biologic agents. Clin Exp Rheumatol 2013;31:S14-18.

25 van der Woude D, Young A, Jayakumar $K$, et al. Prevalence of and predictive factors for sustained disease-modifying antirheumatic drug-free remission in rheumatoid arthritis: results from two large early arthritis cohorts. Arthritis Rheum 2009;60:2262-71.

26 Klarenbeek NB, van der Kooij SM, Guler-Yuksel M, et al. Discontinuing treatment in patients with rheumatoid arthritis in sustained clinical remission: exploratory analyses from the BeSt study. Ann Rheum Dis 2011;70:315-19.

27 van der Kooij SM, Goekoop-Ruiterman YP, de Vries-Bouwstra JK, et al. Drug-free remission, functioning and radiographic damage after 4 years of response-driven treatment in patients with recent-onset rheumatoid arthritis. Ann Rheum Dis 2009;68:914-21

28 Rantapaa-Dahlqvist S, de Jong BA, Berglin E, et al. Antibodies against cyclic citrullinated peptide and IgA rheumatoid factor predict the development of rheumatoid arthritis. Arthritis Rheum 2003;48:2741-9.

29 van der Helm-van Mil AH, Verpoort KN, et al. Antibodies to citrullinated proteins and differences in clinical progression of rheumatoid arthritis. Arthritis Res Ther 2005; 7:R949-958.

30 Harre U, Georgess D, Bang H, et al. Induction of osteoclastogenesis and bone loss by human autoantibodies against citrullinated vimentin. J Clin Invest 2012;122:1791-802.

31 Kleyer A, Finzel S, Rech J, et al. Bone loss before the clinical onset of rheumatoid arthritis in subjects with anticitrullinated protein antibodies. Ann Rheum Dis 2014;73:854-60.

32 Demoruelle MK, Weisman MH, Simonian PL, et al. Brief report: airways abnormalities and rheumatoid arthritis-related autoantibodies in subjects without arthritis: early injury or initiating site of autoimmunity? Arthritis Rheum 2012;64:1756-61. 


\section{ARD}

Relapse rates in patients with rheumatoid arthritis in stable remission tapering or stopping antirheumatic therapy: interim results from the prospective randomised controlled RETRO study

Judith Haschka, Matthias Englbrecht, Axel J Hueber, Bernhard Manger, Arnd Kleyer, Michaela Reiser, Stephanie Finzel, Hans-Peter Tony, Stefan Kleinert, Martin Feuchtenberger, Martin Fleck, Karin Manger, Wolfgang Ochs, Matthias Schmitt-Haendle, Joerg Wendler, Florian Schuch, Monika Ronneberger, Hanns-Martin Lorenz, Hubert Nuesslein, Rieke Alten, Winfried Demary, Joerg Henes, Georg Schett and Juergen Rech

Ann Rheum Dis2016 75: 45-51 originally published online February 6, 2015

doi: 10.1136/annrheumdis-2014-206439

Updated information and services can be found at:

http://ard.bmj.com/content/75/1/45

These include:

Supplementary Supplementary material can be found at:

Material http://ard.bmj.com/content/suppl/2015/02/06/annrheumdis-2014-2064 39.DC1

References This article cites 32 articles, 15 of which you can access for free at: http://ard.bmj.com/content/75/1/45\#ref-list-1

Email alerting service

Receive free email alerts when new articles cite this article. Sign up in the box at the top right corner of the online article.

Topic Articles on similar topics can be found in the following collections Collections

Connective tissue disease (4253)

Degenerative joint disease (4641)

Immunology (including allergy) (5144)

Musculoskeletal syndromes (4951)

Rheumatoid arthritis (3258)

Biological agents (545)

Drugs: musculoskeletal and joint diseases (700)

Epidemiology (1407)

To request permissions go to:

http://group.bmj.com/group/rights-licensing/permissions

To order reprints go to:

http://journals.bmj.com/cgi/reprintform

To subscribe to BMJ go to:

http://group.bmj.com/subscribe/ 


\section{Notes}

To request permissions go to:

http://group.bmj.com/group/rights-licensing/permissions

To order reprints go to:

http://journals.bmj.com/cgi/reprintform

To subscribe to BMJ go to:

http://group.bmj.com/subscribe/ 